\title{
To bed or not to bed: the sleep question?
}

\author{
Chris Brown (10, ${ }^{1}$ Tarig Abdelrahman, ${ }^{1}$ Wyn Lewis, ${ }^{1}$ John Pollitt, ${ }^{1}$ Richard Egan, ${ }^{2}$ on \\ behalf of the members of the Welsh Surgical Research Initiative
}

\begin{abstract}
${ }^{1}$ School of Surgery, Cardiff University School of Postgraduate Medical and Dental Education, Cardiff, UK ${ }^{2}$ Department of Surgery, Morriston Hospital, Swansea, United Kingdom
\end{abstract}

\section{Correspondence to} Dr Chris Brown, Cardiff University School of Postgraduate Medical and Dental Education, Cardiff CF144XW, UK; chris brown87@doctors.org.uk

Received 12 April 2018 Revised 12 August 2019 Accepted 26 November 2019

(c) Author(s) (or their employer(s)) 2019. No commercial re-use. See rights and permissions. Published by BMJ.

\section{To cite: Brown C,} Abdelrahman $T$, Lewis $W$ et al. Postgrad Med J 2020:96:520-524.

\begin{abstract}
Background Sleep deprivation and fatigue from long-shift work impacts doctors' personal safety, inhibits cognitive performance and risks clinical error. The aim of this study was to assess the sleep quality of surgical trainees participating in European Working Time Directive-compliant training rotations within a UK deanery.
\end{abstract}

Methods A trainee cohort numbering 38 (21 core, 17 higher surgical trainees, 29 men and 9 women, median age 31 (25-44 years)) completed a sleep diary over 30 days using the Sleep Time (Azumio) smartphone application and triangulated with on-call rosters to identify shift patterns. The primary outcome measure was sleep quality related to rostered clinical duties.

Results Consecutive 1152 individual sleep episodes were recorded. The median time asleep (hours:min) was $6: 29$ (5:27-7:19); the median sleep efficiency was $86 \%$ (80\%-93\%); the median light sleep (hours:min) was 2:50 (1:50-3:49); and the median rapid eye movement (REM) sleep (hours:min) was 3:20 (2:37-4:07). Significant adverse sleep profiles were observed in trainees undertaking emergency on-call duty when compared with elective (non-on-call) duty; the median time asleep (hours:min) $5: 49$ vs $6: 43(p<0.001)$; the median sleep efficiency was $85 \%$ vs $87 \%(p<0.001)$; the median light sleep (hours:min) was 2:16 vs 2:58 ( $p<0.001)$; and REM sleep (hours:min) was $2: 57$ vs 3:27 ( $p<0.001)$. Recovery of sleep duration, efficiency and quality necessitated five full days of time.

Conclusion Surgical emergency on-call duty adversely influences sleep quality. Proper consideration of fail-safe rota design, prioritising sleep hygiene, recovery and wellbeing, allied to robust patient safety and quality of care should be made a priority.

\section{INTRODUCTION}

Nothing declares first-world economy status more than round-the-clock access to essential services. Markets driven by banking and commerce have little regard for traditional working hours, nowhere more so than healthcare, currently in particular focus, given the push and promise of 7-day universal services for all. Sleeping at night is no longer the default option, and many decide that to permit other pursuits, they can survive with less sleep, but at what cost?

A litany of disasters with global reach has been blamed on sleep deprivation; nuclear accidents at Three Mile Island in 1979 and at Chernobyl in 1986 each took place at night and involved serious judgement errors by tired operators. When the Space Shuttle exploded in 1986, killing all aboard, the NASA launch authorisation managers who sanctioned take-off, despite poor weather, had little sleep the night before. ${ }^{1}$ On a more everyday level, road traffic accidents more likely involve sleepy drivers, ${ }^{2}$ and the US National Commission on Sleep Disorders reported driver fatigue contributed to over half of all accidents. ${ }^{3}$ Moreover, even if sleep deprivation does not result in death or injury, clinical studies report that it sways performance severely, which ought to worry the chief of every sleep sick employee. ${ }^{4}$ Yet data in the fields of medicine, anaesthesia and surgery are thin, which is surprising when the stakes are so high in terms of risk to life. Fatigue and sleep deprivation, associated with shift work and long working hours impact on doctors' personal safety, increasing the likelihood of occupational accidents. Moreover, it also risks patient safety and quality of clinical care because poor cognitive performance inevitably results in medical error. ${ }^{5}$ Legal restrictions governing doctors' working conditions were first introduced in 1989, in US New York State following the death of 18-year-old Libby Zion, who was attended by doctors on 36-hour shifts. Yet not until 2003 were US trainees limited by law to a universal 80-hour week. ${ }^{6}$ The European Working Time Directive (EWTD) legislation, enshrined in UK law as the Working Time Regulations 1998 and in force in the UK since 2009, provides a number of protections for doctors. These include an average limit of 48 hours a week, a daily minimum period of 11 hours continuous, a day off each week, a 20 min rest break every 6 hours and a limit of 8 hours worked in every 24-hour period for night work, and mandates employers to keep records of hours worked. ${ }^{7}$ The EWTD confers significant benefits on doctors in terms of protecting their health and safety and that of their patients. Limiting working hours can help to reduce the likelihood of tiredness and therefore improve the service quality delivered to patients. When doctors are well rested and alert, thanks to improved work-life balance, safety risks both on and off duty are also reduced. ${ }^{3}$

The objective of this study was to assess sleep behaviour in a cohort of surgical trainees working in a single UK Deanery School of Surgery, compliant with EWTD legislation, to determine the influence of emergency on-call duties on sleep duration and quality.

\section{METHOD}

Nationally appointed core and higher general surgical trainees within a single UK deanery were recruited voluntarily by means of email invitation. No selection criteria were employed other than enrolment on a core or higher surgical training (HST) scheme and possession of a smartphone. 
Table 1 Comparison of sleep parameters related to grade

\begin{tabular}{|c|c|c|c|c|}
\hline & Total & CST $(n=21)$ & HST $(n=17)$ & $P$ value \\
\hline Time in bed (hh:mm) & $7: 29(6: 36-8: 15)$ & $7: 20(6: 32-8: 11)$ & $7: 38(6: 46-8: 21)$ & 0.003 \\
\hline Efficiency (\%) & $86(80-93)$ & 86 (79-94) & $86(80-93)$ & 0.621 \\
\hline Time asleep (hh:mm) & $6: 29(5: 27-7: 19)$ & $6: 22(5: 19-7: 17)$ & $6: 38(5: 40-7: 23)$ & 0.018 \\
\hline Light sleep (hh:mm) & $2: 50(1: 50-3: 49)$ & $2: 47(1: 44-3: 46)$ & $2: 52(1: 52-3: 55)$ & 0.124 \\
\hline Deep/REM sleep (hh:mm) & $3: 20(2: 37-4: 07)$ & $3: 17(2: 38-4: 10)$ & $3: 25$ (2:37-4:06) & 0.901 \\
\hline
\end{tabular}

All values are median (IQR) unless specified.

CST, core surgical training; hh, hours; HST, higher surgical training; mm, minutes; REM, rapid eye movement.

Sleep-tracking applications are increasingly popular as a method of monitoring sleep patterns, taking advantage of the movement tracking technology capabilities of contemporary smartphones using an integrated actigraph. The five most popular sleeptracking applications were tested by two authors (RE and $\mathrm{CB}$ ), and Sleep Time by Azumio ${ }^{8}$ was selected for its perceived userfriendly interface, ease of data collection and compatibility with multiple smartphone operating systems. Basic demographic details of participants were recorded, including age, gender, training grade and current hospital of work. Using the Sleep Time application, participants were required to track their sleep each night for the calendar month of November 2017, including weekends and annual and study leaves. The application instructed participants to place their smartphone on their pillow or mattress each night to enable the actigraph to record all movements during sleep and differentiate between different physiological sleep cycles. Using the integrated actigraph, the sleep application can differentiate broadly between light sleep and deep/rapid eye movement (REM) sleep, as well as calculate overall sleep time and efficiency. Participants were also asked to record their shift patterns for the day before each recorded sleep episode (either normal working day, on-call day shift or on-call night shift). After each recorded sleep episode, the summary report from the Sleep Time application was forwarded electronically to the senior author (RE) for amalgamation. Following completion of the data submission period, all participants were asked to estimate their average nightly sleep time for comparison with the objective data.

\section{Statistical analysis}

Inferential statistics appropriate for non-parametric data were conducted using IBM SPSS Statistics V.23. The primary outcome measure was the effect of on-call duties on sleep quality parameters, including duration of time in bed, time asleep, sleep efficiency (the ratio of the total time spent asleep (total sleep time) in a night compared with the total amount of time spent in bed), duration of light sleep and duration of deep/REM sleep. Secondary outcome measures included comparison of sleep parameters related to gender and trainee grade. There we no data exclusions. Grouped differences were analysed using paired/independent sample t-tests.

\section{RESULTS}

Thirty-eight volunteer trainees were included in the analysis, comprising nine women (23.7\%) and 29 men $(76.3 \%)$ of median age 31 years (range 25-44). Twenty-one were core surgical trainees and 17 higher surgical trainees. Thirty-four participants (89\%) submitted complete, consecutive data for the whole calendar month, and 1152 individual sleep episodes were recorded overall. Participant duties recorded during the data collection period comprised 121 night shifts (20:00-08:00), 149 on-call days (08:00-20:00), 399 normal working days (08:00-17:00) and 483 non-working days (inclusive of compensatory rest and annual and study leaves).

Sleep parameters related to grade and gender are shown in tables 1 and 2.

The comparison of sleep parameters collected from the sleep episode after completion of on-call duty (either night or day shift) and while not on call (normal working day, day off and annual or study leave) is shown in table 3 .

The recovery of overall time asleep after on-call night-shift duties is shown in figure 1. Figure 2 shows the recovery of sleep efficiency after on-call night-shift duties.

Weak correlation was observed between self-reported time asleep (hours:min) (median 7:00, range 5:00-8:00) and actual recorded time asleep (hours:min) (median 6:29, range 5:03$87: 27$ ) (Pearson's $r=0.313, p=0.010$ ), suggesting a general overestimation of nightly sleep duration by trainees.

\section{DISCUSSION}

It is widely assumed and confidently asserted at scientific meetings that working time regulations have solved the age-old problem of the sleep-deprived medical professional. Such sweeping assumptions are dismissed by the results of this study, the first to objectively measure relative sleep patterns and quality in a spectral cohort of surgeons in training. Going by the numbers, despite doctors' altered working patterns after EWTD introduction, the degrading effect of sleep deprivation was clearly evident, implying doctors continue to experience considerable work-derived fatigue. ${ }^{9}$ The recommended sleep time for a typical adult is 7-9 hours, with very good sleep efficiency defined as being $\geq 90 \%$ and an average night's sleep comprising 55\% light sleep, 20\% deep sleep and 25\%

Table 2 Comparison of sleep parameters related to gender

\begin{tabular}{lcccc}
\hline & Total & Male $(\mathrm{n}=29)$ & Female $(\mathrm{n}=9)$ & P value \\
\hline Time in bed (hh:mm) & $7: 29(6: 36-8: 15)$ & $7: 23(6: 30-8: 10)$ & $7: 43(7: 00-8: 34)$ & $<5(80-90)$ \\
Efficiency (\%) & $86(80-93)$ & $87(80-94)$ & $6: 43(5: 43-7: 30)$ & 0.031 \\
Time asleep (hh:mm) & $6: 29(5: 27-7: 19)$ & $6: 24(5: 18-7: 15)$ & $2: 33(1: 20-3: 40)$ & $<: 401$ \\
Light sleep (hh:mm) & $2: 50(1: 50-3: 49)$ & $2: 55(1: 57-3: 52)$ & $3: 56-4: 45)$ & $<0.001$ \\
Deep/REM sleep (hh:mm) & $3: 20(2: 37-4: 07)$ & $3: 09(2: 32-3: 54)$ & $<0.001$ \\
\hline
\end{tabular}

All values are median (IQR) unless specified.

hh, hours; mm, minutes; REM, rapid eye movement. 
Table 3 Comparison of sleep parameters obtained during the sleep episode following an on-call duty (combined night [20:00-08:00) and day shifts (08:00-20:00) and while not on-call

\begin{tabular}{lcccc}
\hline & Total & On-call duty (night/day shifts) & Not on call (NWD/DO/AL/SL) & P value \\
\hline Time in bed (hh:mm) & $7: 29(6: 36-8: 15)$ & $6: 47(5: 20-7: 36)$ & $7: 37(6: 53-8: 26)$ & $<0.001$ \\
Efficiency (\%) & $86(80-93)$ & $85(78-91)$ & $87(80-94)$ & 0.001 \\
Time asleep (hh:mm) & $6: 29(5: 27-7: 19)$ & $5: 49(4: 15-6: 38)$ & $6: 43(5: 44-7: 29)$ & $<0.001$ \\
Light sleep (hh:mm) & $2: 50(1: 50-3: 49)$ & $2: 16(1: 20-3: 16)$ & $3: 58(2: 03-3: 57)$ & $<0.001$ \\
Deep sleep (hh:mm) & $3: 20(2: 37-4: 07)$ & $2: 57(2: 10-3: 40)$ & $3: 45-4: 13)$ & $<0.001$ \\
\hline
\end{tabular}

All values are median (IQR) unless specified.

$\mathrm{AL}$, annual leave; DO, day off; hh, hours; mm, minutes; NWD, normal working day; SL, study leave.

REM. $^{10} 11$ The principal finding was that on-call duties were associated with significant adverse consequences related to all major sleep parameters, including total sleep duration (14\% less), time in bed (11\% less), REM/deep sleep (24\% less) and overall sleep efficiency ( $4 \%$ poorer). These findings were more marked in core surgical training than HST, with the latter cohort achieving a significantly longer overall time in bed and time asleep. The reason for this is not apparent from the data collated, but it could arguably be postulated that higher surgical trainees will have had more experience of night-shift work and are therefore more likely to have developed better sleep hygiene practices than more junior core surgical trainee peers. Gender-related differences were also apparent; women's time asleep was 5\% longer, but sleep efficiency was $2 \%$ poorer and deep sleep was $20 \%$ longer from those recorded in men. Night shifts were associated with particularly detrimental features, and on average, no less than 5 days were required to recover to prenight-shift levels of sleep duration, efficiency and quality.

Sleep quality is a term widely used by researchers, clinicians and the public alike yet lacks consensus regarding a precise definition. Good sleep quality is well recognised to predict physical and mental health, well-being and overall vitality. Short sleep latencies and less wakening, equating to better sleep efficiency (>85\%), have all been described as indices of good sleep quality, regardless of age. Current recommended adult sleep duration for adults is 7-9 hours, which allegedly two-thirds of all adults in developed nations fail to enjoy ${ }^{11}$ and the results of this study corroborate. Overall monthly sleep times averaged of 6 hours $9 \mathrm{~min}$, which then deteriorates to a mere 5 hours $49 \mathrm{~min}$ after on-call shifts. Moreover, the correlation between actual and

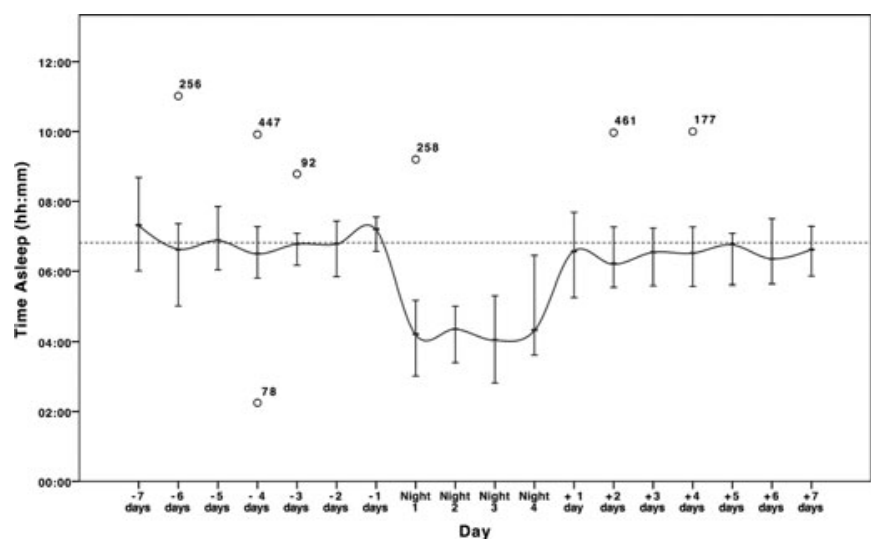

Figure 1 Boxplot showing recovery of sleep time for the 7 days before and 7 days after a set of four night shifts. Dashed line demonstrates median sleep efficiency for the 7 days before starting night shifts. subjective times asleep implies a significant overestimation in the perceived time asleep (Pearson's $\mathrm{r}=0.313, \mathrm{p}=0.010$ ).

Every $90 \mathrm{~min}$ during a typical night, the brain moves through a cycle of two completely different types of sleep, characterised through polysomnography by their defining ocular features: non-rapid eye movement (NREM) sleep (comprising stages N1 and N2 (light) and N3 (deep) sleep) and REM sleep. The ratio of NREM to REM sleep changes dramatically during the night, with the majority of early sleep cycles consisting of deep NREM sleep, and later cycles rich in REM sleep. No consensus exists regarding why sleep cycles occur in this asymmetrical pattern, though one theory contends that it is necessary to remodel and update neural circuits in order to manage finite brain storage. NREM sleep's key function is thought to be removal of unnecessary neural connections, followed later by REM sleep's dream stage, which plays a role in refining neural connections. MRI studies have reported that key emotion and memory-related brain structures are reactivated during REM sleep, and another key theory regarding REM sleep's function relates to memory processing, in particular if traumatic or emotional in nature. ${ }^{11}$ The significant $24 \%$ fall in deep/REM sleep observed after trainees had performed on-call shift duties is consequently particularly troubling and apparently takes some 5 days or 124 hours to recover. Fatigue derived from sleep deprivation is associated with a myriad of effects related not only to personal well being but also to issues of patient safety and quality of care. A recent survey of UK anaesthetic trainees reported that $85 \%$ of respondents felt unsafe to drive home following night duty, and an increased risk of accidents has been associated with lack of sleep and shift work. Moreover, 57\% of trainees admitted to having been involved in

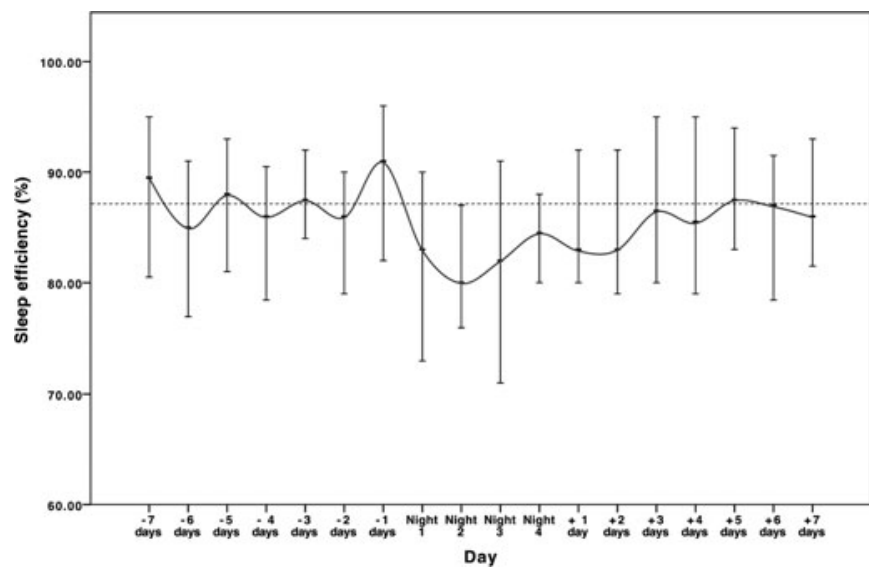

Figure 2 Boxplot showing recovery of sleep efficiency (\%) for the 7 days before and 7 days after a set of four night shifts. Dashed line demonstrates median time asleep for the 7 days before starting night shifts. 
either a road traffic incident or a near miss following a night shift. $^{12} 13$

Paying back sleep debt needs time, with very few interventions reported to influence this process. In the recently published guidance: The Fatigue and Facilities Charter (March 2018), designed to improve awareness of good sleep practices, the British Medical Association outlined a number of proposals aimed at minimising fatigue associated with shift work and improving hospital policy and facilities. Key recommendations included a minimum of 46 hours' rest period following completion of the final night shift, a maximum of seven consecutive shifts with no more than 72 hours duty in a 168 hours' period, the provision of basic education on working nights and sleep hygiene during induction, an accessible 24/7 hospital mess with appropriate rest areas, complimentary sleep facilities for all staff rostered on-call at night, and where possible, appropriate sleep facilities or alternative travel arrangements for doctors who feel unable to travel home after a night shift or a long, late shift due to tiredness. ${ }^{14}$

Reports regarding the effects of fatigue have found that cognitive performance and prospective memory are globally reduced following only 25 hours of sleep deprivation. ${ }^{15}$ This reduction in performance has been equated to the effect of a $0.1 \%(100 \mathrm{mg} / \mathrm{dL})$ blood alcohol content, a finding that would reprimand a physician as unfit to assume clinical duties. ${ }^{16}$ Whether this reduction in cognition translates into a reduction in clinical performance is uncertain, with conflicting results reported in the literature. In a systematic review, Sturm et al reported that only two of five clinical outcome studies demonstrated an increased rate of errors and complications in sleep-deprived surgeons. Studies related to simulation-based outcomes also reported mixed findings, although junior trainees were apparently more at risk of fatigue than their more senior colleagues. ${ }^{17}$ Rothschild et al, in a large single-centre study, including surgical and obstetric procedures, reported twice as many complications after elective operations when the surgeon had performed out-of-hours, night-time surgery, within 6 hours of the elective operation, when compared with surgeons who had performed similar emergency procedures more than 6 hours before the elective operation $(6.2 \%$ vs $3.4 \%$, OR $1.72(1.02-2.89)) .{ }^{18}$

Most evidence linking fatigue to outcomes is based on short periods of sleep deprivation, equating to one missed or broken night of sleep, prior to a predefined event, with little published data assessing the cumulative effect of sleep fatigue. McKenna and Wilkes reported an inverse correlation between performance and sleep deprivation of as little as 2 hours, with progressive performance deterioration associated with increasing sleep debt. This clearly places surgical trainees at risk of suboptimal performance, starting with the first night shift. Furthermore, evidence supporting intershift manoeuvres to improve sleep is thin and limited to sleep hygiene steps such as avoiding caffeine and blue-screen electronics. Most evidence supporting methods to minimise prenight sleep debt is confined to the day prior to night 1 . McKenna and Wilkes report that this is best achieved by allowing unrestricted sleep (ie, no alarm) on mornings prior to starting night shifts, with a supplementary sleep episode of 60-90 min on the afternoon of the same day. ${ }^{19}$ Whether such opportunities are always possible in contemporary surgical training is doubtful, with many using such time to pursue extracurricular activities, cover clinical sessions or complete personal 'life-administration' tasks.

An individual's sleep requirement and tolerance to sleep deprivation has been reported to vary with age, ${ }^{11}$ but evidence supporting specific specialties exhibiting specific sleep patterns or resilience is thin. Woodrow et al reported higher levels of subjective sleepiness by surgical residents when compared with psychiatry and medical residents but also described a hidden culture of surgical residential denial related to sleep deprivation. ${ }^{20}$

This study has a number of inherent potential limitations. Information gathered was dependent on the accuracy and consistency with which trainees recorded data using the application, and placement of smart phones in the recommended position on their pillow or mattress for the duration of each sleep episode. This application has been developed for educational purposes and cannot be compared or used as a substitute for formal sleep medicine evaluation using gold standard, laboratory-based polysomnography. Previous research has demonstrated the application to have high accuracy for sleep-wake detection (85.9\%) but rather poor accuracy in differentiating between phases of sleep (45.9\%). The application also slightly overestimated sleep efficiency by $0.12 \%$ when compared with gold standard, inlaboratory polysomnography $(p=0.962) .{ }^{21}$ This was considered workable, as the primary outcome measures were sleep duration and sleep efficiency rather than an in-depth analysis of sleep phases. A further limitation was the failure to capture unplanned episodes of sleep, which might influence analysis of sleep patterns before night shifts (eg, afternoon napping) and during night duty, although rarely likely in trainee job plans. Night-shift napping may improve alertness and sleep-related performance, but little provision is made to allow night-time sleep, with facilities available in a minority of hospitals in the region. ${ }^{20}{ }^{22}$ In contrast, the study has a number of strengths, representing original work, consisting of a large dataset equivalent to more than 3 years' sleep. Moreover, it has statistical power and has demonstrated the role and cost of effective, efficient and readily available personal wearable technology for large-volume data collection.

In conclusion, the findings of this study suggest that participation in surgical on-call rotas has a negative impact on several parameters associated with sleep quality, with night shifts in particular posing a high-risk. The demands of surgical training produce work-related pressures and emotional stress which, compounded by chronic sleep deprivation, not only affect the well-being of trainees but also likely influence clinical results and threaten patient safety and quality of care. Burnout is becoming increasingly reported among junior doctors, and symptoms akin to post-traumatic stress disorder ${ }^{23}$ allegedly affect as many as two-thirds of UK junior doctors, causing damaged physical or mental health due to intense National Health Service (NHS) work-related physical and emotional burden. ${ }^{24}$ Given the negative impact of long hours on trainees' ability to perform safely, organisations can no longer afford to ignore the influences of hostile training environments and unsafe working patterns. Proper consideration of fail-safe rota design, prioritising trainee recovery and well-being over service provision, must be a priority.

\section{Main messages}

- On-call duties were associated with significant adverse consequences related to all major sleep parameters, including total sleep duration (14\% less), time in bed (11\% less), REM/deep sleep (24\% less) and overall sleep efficiency ( $4 \%$ poorer).

- Night shifts were particularly detrimental, requiring on average 5 days' recovery to prenight-shift levels of sleep duration, efficiency and quality.

- Proper consideration of fail-safe rota design, prioritising trainee recovery and well-being over service provision, must be a priority. 


\section{Current research questions}

What is the effect of sleep deprivation on doctors' training outcomes and patient safety and quality of care?

- What is the ideal, pragmatic rota that allows complete recovery after sleep deprivation following on-call duty and night-shift work?

- Is there and should there be a role for formal polysomnography for in-depth assessment of junior doctor sleep cycles?

\section{What is already known}

- Contemporary surgical training in the UK typically involves highintensity, time-pressured working patterns which can lead to sleep deprivation and fatigue.

- Fatigue and sleep deprivation, associated with long working hours and shift work, impacts on doctors' personal safety, increasing the likelihood of occupational accidents, road traffic crashes and needle stick injuries.

It also increases risks to patient safety through clinical errors.

\section{Twitter Chris Brown @Dr_Chris_Brown}

Collaborators Sabria Abdulal, Jake Ahmed, Adnan Ahmad, Nicola Allen, James Ansell, Stefan Arnaudov, Rachael Barnett, Andrew Beamish, David Bosanquet, David Chan, Susan Chandler, Madlen Dewi, Sam Dwalesena, Andrew Gardner, Gianluca Gonzi, Rhiannon Harries, Luke Hopkins, Michael Hopkins, Osian James, Ali Jawad, Dylan Jones, Huw Jones, Rod Jones, Oliver Luton, Christopher Marusza, Harshul Measuria, Samir Mehta, Jack Pearce, Tito Petralia, Arfon Powell, Anna PowellChandler, Nicola Reeves, David Selwyn, Zoe Seymour, Ronak Ved.

Contributors CB: data acquisition, drafting, final approval and submission. TA: data analysis, drafting and final approval. WL: data interpretation, critical revision and final approval. JP: data interpretation, critical revision and final approval. RE: study conception, drafting and final approval prior to submission. All authors agree to be accountable for all aspects of the work in ensuring that questions related to the accuracy or integrity of any part of the work are appropriately investigated and resolved. Welsh Surgical Research Initiative: data collection.

Funding The authors have not declared a specific grant for this research from any funding agency in the public, commercial or not-for-profit sectors.

Competing interests None declared.

Patient consent for publication Not required.

Ethics approval Ethical approval for this study was granted by the NHS Research Authority and Health and Care Research Wales (IRAS 254658).

Provenance and peer review Not commissioned; externally peer reviewed.

Data availability statement Data are available upon reasonable request.

ORCID iD

Chris Brown http://orcid.org/0000-0003-1490-6287

\section{REFERENCES}

1 Mitler MM, Carskadon MA, Czeisler CA, et al. Catastrophes, sleep, and public policy: consensus report. Sleep 1988;11:100-9.

2 Connor J, Norton R, Ameratunga $S$, et al. Driver sleepiness and risk of serious injury to car occupants: population based case control study. BMJ 2002;324:1125.

3 pp.Leger D. The cost of sleep-related accidents: a report for the National Commission on sleep disorders research. Sleep 1994;17:84-93.

4 The Economist. And so to bed. [online], 2002. Available: http://www.economist.com /node/1487570 [Accessed 5 Dec. 2017].

5 Bma.org.uk. BMA - Fatigue and sleep deprivation. [online], 2019. Available: https:// www.bma.org.uk/collective-voice/policy-and-research/education-training-andworkforce/fatigue-and-sleep-deprivation [Accessed 23 Feb 2019].

6 Zinner MJ, Fresichlag JA. Surgeons, sleep, and patient safety. JAMA 2013;310:1807-8

7 Directive 2003/88/EC of the European Parliament and of the Council of 4 November 2003 concerning certain aspects of the organisation of working time. Off J L 2003:299:9-19.

8 Sleep Time, Azumio Inc. TM, 230 S California Avenue, Ste 212, Palo Alto, CA94306, Available: http://www.azumio.com

9 Bodkin $\mathrm{H}$. Junior doctors crashing after exhausting hospital night shifts. [online] The Telegraph. Available: http://www.telegraph.co.uk/news/2017/07/06/junior-doctorscrashing-exhausting-hospital-night-shifts/ [Accessed 13 Feb 2018].

10 Shrivastava $D$, Jung $S$, Saadat M, et al. How to interpret the results of a sleep study. J Community Hosp Intern Med Perspect 2014;4.

11 Walker M. Why we sleep: the new science of sleep and dreams. Allen Lane (Penguin Random House, UK)., 2017. ISBN: 978-0-141-98377-6.

12 pp.McClelland L, Holland J, Lomas J-P, et al. A national survey of the effects of fatigue on trainees in anaesthesia in the UK. Anaesthesia 2017:72:1069-77.

13 Kecklund G, Axelsson J. Health consequences of shift work and insufficient sleep. BMJ 2016;355:i5210.

14 Bma.org.uk. BMA - Fatigue and facilities charter. [online], 2018. Available: https://w ww.bma.org.uk/advice/employment/working-hours/fatigue-and-facilities-charter [Accessed 27 Mar 2018].

15 Grundgeiger T, Bayen UJ, Horn SS. Effects of sleep deprivation on prospective memory. Memory 2014;22:679-86.

16 Dawson D, Reid K. Fatigue, alcohol and performance impairment. Nature 1997;388:235.

17 Sturm L, Dawson D, Vaughan R, et al. Effects of fatigue on surgeon performance and surgical outcomes: a systematic review. ANZ J Surg 2011;81:502-9.

18 Rothschild JM, Keohane CA, Rogers $S$, et al. Risks of complications by attending physicians after performing nighttime procedures. JAMA 2009;302:1565-72.

19 McKenna H, Wilkes M. Optimising sleep for night shifts. BMJ 2018;360:j5637.

20 Woodrow SI, Park J, Murray BJ, et al. Differences in the perceived impact of sleep deprivation among surgical and non-surgical residents. Med Educ 2008:42:459-67.

21 Bhat S, Ferraris A, Gupta D, et al. Is there a clinical role for smartphone sleep Apps? comparison of sleep cycle detection by a smartphone application to polysomnography. J Clin Sleep Med 2015;11:709-15.

22 Ruggiero JS, Redeker NS. Effects of napping on sleepiness and sleep-related performance deficits in night-shift workers: a systematic review. Biol Res Nurs 2014;16:134-42.

23 Thompson CV, Naumann DN, Fellows JL, et al. Post-traumatic stress disorder amongst surgical trainees: an unrecognised risk? Surgeon 2017;15:123-30.

24 Campbell D. Two-thirds of young hospital doctors under serious stress, survey reveals. The Guardian. [online], 2017. Available: http://www.theguardian.com /uk-news/2017/feb/11/stressed-out-doctors-anaesthetists-survey-nhs [Accessed 31 May 2017]. 